\title{
Wavelet and Neural Structure: A New Tool for Diagnostic of Power System Disturbances
}

\author{
Dolores Borrás, M. Castilla, Member, IEEE, Narciso Moreno, and J. C. Montaño, Senior Member, IEEE
}

\begin{abstract}
The Fourier transform can be used for analysis of nonstationary signals, but the Fourier spectrum does not provide any time-domain information about the signal. When the time localization of the spectral components is needed, a wavelet transform giving the time-frequency representation of the signal must be used. In this paper, using wavelet analysis and neural systems as a new tool for the analysis of power system disturbances, disturbances are automatically detected, compacted, and classified. An example showing the potential of these techniques for diagnosis of actual power system disturbances is presented.
\end{abstract}

Index Terms-Harmonic distortion, neural networks, signal analysis, transforms, wavelets.

\section{INTRODUCTION}

$\mathbf{I}$ N ORDER to determine the sources and causes of harmonic distortion of the voltage signal delivered by utilities, one must be able to detect and localize those disturbances and classify the different types. Software procedures applying the fast Fourier transform (FFT) have been developed for this purpose [1], but due to the great amount of stored data and the time required for processing, such procedure is slow and not very efficient.

Continuous and discrete wavelet transforms (CWTs and DWTs) have been used in analysis of nonstationary signals, and several recent papers, such as [2] and [3], have proposed the use of wavelets for power systems analysis. Wavelet transforms are mathematical tools with powerful structure and enormous freedom that decompose a given signal into several scales at different levels of resolution. At each scale, the WT coefficients corresponding to a given disturbance are larger than those not corresponding to such disturbance. Thus, related coefficients are kept, while others not related to the disturbance are discarded. As a consequence, data could be reduced considerably in number with very little loss of information.

D. Borrás, M. Castilla, and N. Moreno are with the Department of Electrical Engineering, Universidad de Sevilla, 41011 Seville, Spain (e-mail: narciso-ma@us.es).

J. C. Montaño is with Consejo Superior de Investigaciones Científicas, Seville, Spain (e-mail: montano@irnase.csic.es).
In this work, a WT approach is proposed to detect and classify various types of power systems disturbance. The method selects the most suitable type of wavelet and applies the DWT. The reconstruction process is thereby obtained both with and without the disturbed signal, using a reduced number of coefficients. The algorithm of coefficient filtering to compress the signal is based on the procedures described in [4]. A neural network structure may be used to classify typical disturbances found in power systems.

Definitions and concepts of WT are introduced in Section II. The proposed method is described in Section III-A. Finally, results of simulation are given in Section III-B and conclusions in Section IV.

\section{WAVELET THEORY}

Wavelets are functions that satisfy certain mathematical requirements and are used in representing data or other functions. This idea is not new. Approximation using superposition of functions has existed since the early 19th century with Fourier analysis. The Fourier transform (FT) uses basis functions (sines and cosines) to analyze and reconstruct a function. The wavelet approach is more suitable than the Fourier one, especially when signals are nonstationary. Wavelet algorithms process data at different scale or resolution. In wavelet analysis, the scale that we use to look at data plays a special role. A basis function varies in scale by chopping up the same function or data space using different scale sizes. Various wavelets are obtained from a single wavelet $\Psi(t)$ (mother wavelet) by scaling and shifting operations.

A signal or function $f(t)$ can often be better analyzed or processed if expressed as a linear decomposition by

$$
f(t)=\sum c_{l} \Psi_{l}(t)
$$

where $l$ is an integer index, $c_{l}$ are the real coefficients, and $\Psi_{l}(t)$ is a set of functions if the expansion (1) is unique. If the basis is orthogonal, it should also satisfy

$$
\left\langle\Psi_{k}(t), \Psi_{l}(t)\right\rangle=0, \quad k \neq l
$$

where \langle\rangle is the inner product.

For the wavelet expansion, (1) is expressed as

$$
f(t)=\sum_{j} \sum_{k} c_{j, k} \Psi_{j, k}(t)
$$




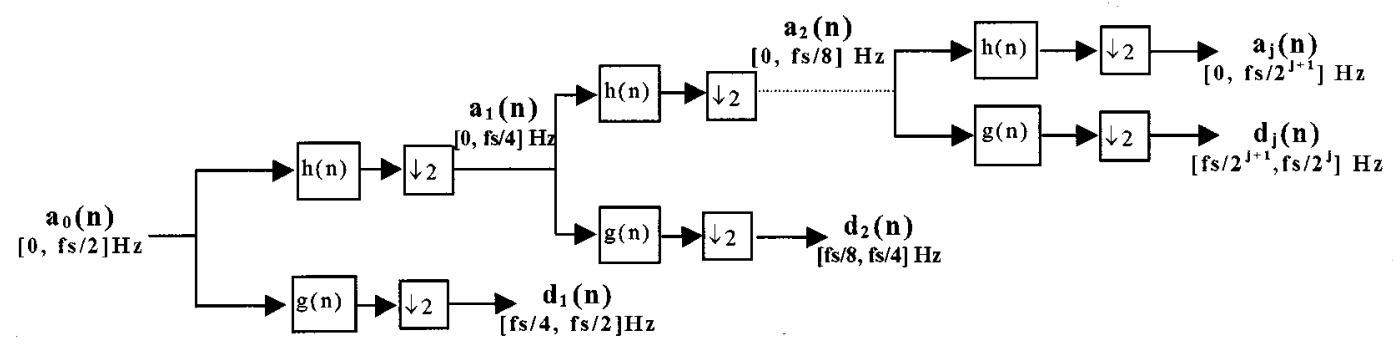

Fig. 1. Multiresolution signal decomposition.

where $j$ and $k$ are integer indexes and $\Psi_{j, k}(t)$ are the wavelet functions that form an orthogonal basis. The set of coefficients $c_{j, k}$ is called $D W T$ of $f(t)$ function, and can be calculated by

$$
c_{j, k}=\int f(t) \Psi_{j, k}(t) d t=\left\langle f(t), \Psi_{j, k}(t)\right\rangle .
$$

The DWT is implemented using a multiresolution signal decomposition algorithm [5], [6] to decompose a given signal into scales with different time and frequency resolution. In this sense, a recorder-digitized function $a_{0}(n)$, which is a sampled signal of $f(t)$, is decomposed into its smoothed version $a_{1}(n)$ (containing low-frequency components), and detailed version $d_{1}(n)$ (containing higher-frequency components), using filters $h(n)$ and $g(n)$, respectively. This is a first-scale decomposition. The next higher scale decomposition is now based on signal $a_{1}(n)$ and so on (Fig. 1). The analysis filter bank divides the spectrum into octave bands. The cutoff frequency for a given level $j$ is found by

$$
f c=\frac{f s}{2^{j+1}}
$$

where $f_{s}$ is the sampling frequency.

After a looked selection, in the present work, Daubechies's wavelets are chosen because they provide a much more effective analysis than that obtained with other wavelets (Haar, Coifman, etc.). This choice is fundamental in order to evaluate wavelets' suitability for signal analysis and data compression by the proposed method.

\section{DETECTION, LOCALIZATION, AND DISCRIMINATION OF POWER SYSTEM DisTURBANCES}

\section{A. Method}

In this section, we propose an effective method (Fig. 2) to analyze and discriminate power system disturbances using wavelet decomposition. The DWT (Daubechies family Db4) is applied to a digitized function with $N$ samples, getting signals $a_{j}(n)$ and $d_{j}(n)$, according to [8], where $j$ are the index levels. The number of samples equals two to the power of the number of levels. The conceptual block diagram is represented in Fig. 3.

1) Detection and Compression: The information about the position and length of a power system disturbance is obtained from the detail coefficients $d_{1}$. This signal $d_{1}(n)$ is nonzero when a disturbance exists.

After detection of the disturbance, a parallel process begins, on the one hand to compress the input signal, and on the other to classify the disturbance, based upon the wavelet decomposition.

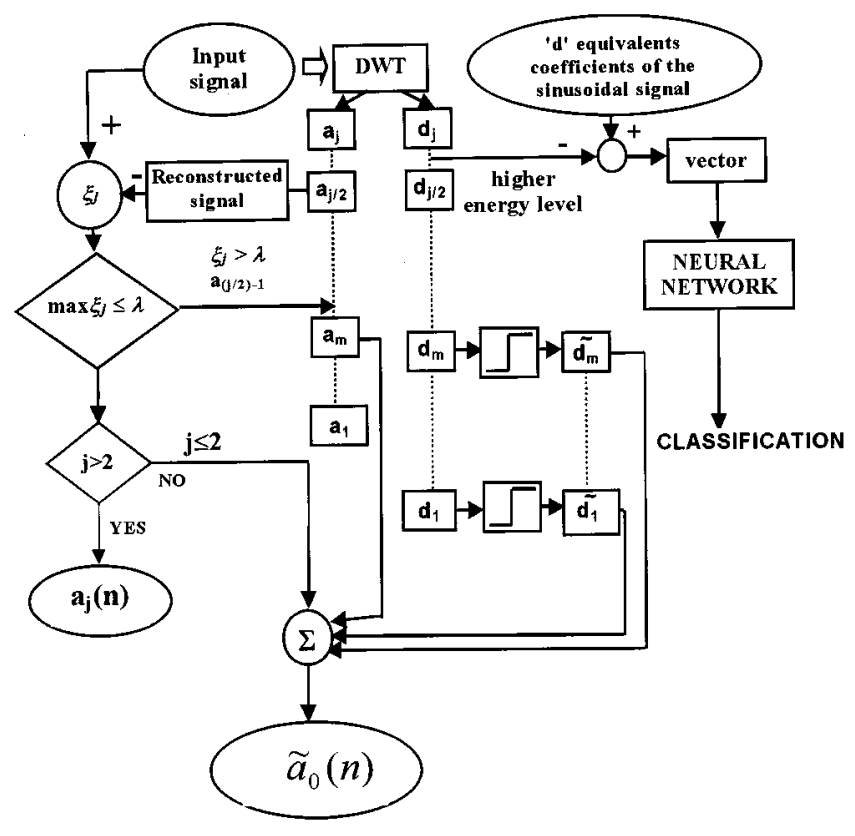

Fig. 2. Flow chart of the proposed method.

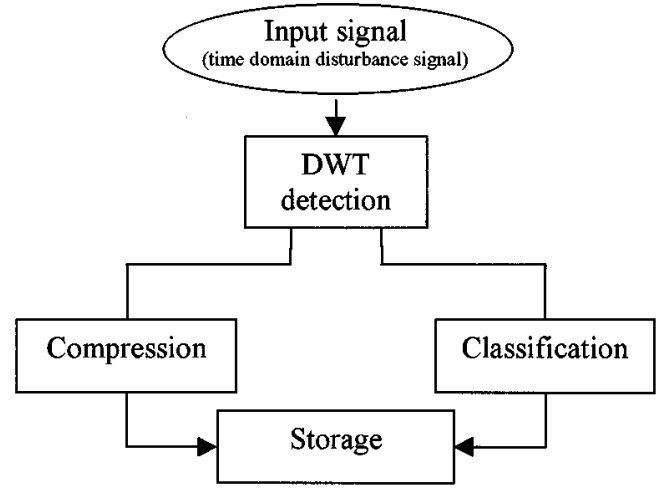

Fig. 3. Conceptual block diagram.

In a first step, an umbral process begins, based at maximum absolute value of original signal. The initial threshold is expressed by

$$
\lambda=\eta \cdot \max \left|a_{0}(n)\right|
$$

where $a_{0}(n)$ is the sampled input signal and $\eta$ is a parameter that varies between $0.01-0.1$. Thus, $\eta=0.03$, the threshold $\lambda$ is $3 \%$ of the maximum value of the input signal. A process of comparison between the input signal and the reconstructed signal $a_{j}(n)$ 
begins. Starting at middle level $j$, comparison between signals $a_{j}(n)$ and input signal $a_{0}(n)$ gives the error signal

$$
\xi_{j}=a_{0}(n)-a_{j}(n), \quad j=1,2, \cdots, \log _{2} N .
$$

The absolute maximum value of $\xi_{j}$ is compared to a fixed threshold $\lambda$. If the magnitude of the error signal is less than $\lambda$, then the resulting signal (reconstructed signal) is the estimated function $\tilde{a}_{0}(n)$, i.e.,

$$
\tilde{a}_{0}(n)=a_{j}(n), \quad \text { if } \max \left|\xi_{j}\right| \leq \lambda .
$$

One of the goals of the present work is to reach a high compression ratio

$$
\tau=\frac{\text { compressed signal data }}{\text { original signal samples }} \cdot 100
$$

for expressing the minimum amount of data, i.e., the essential information, necessary for recovering the input signal $(\tau<$ $20 \%$ ).

If $j \leq 2$, then $\tau>20 \%$ and the algorithm begins a second step to determine a new estimated function without ignoring the $d_{j, k}$ coefficients, which at these levels contain useful information about the disturbance. In this case, for a compromise between a good approach and a high rate of compression, we examine the hard-thresholding estimator. The absolute values of all wavelet coefficients are compared to a threshold $\lambda_{j}$. If the magnitude of the coefficient is less than $\lambda_{j}$, the new coefficient value is zero, otherwise the coefficient value is $d_{j, k}$, i.e.,

$$
\tilde{d}_{j, k}= \begin{cases}d_{j, k}, & \text { if }\left|d_{j, k}\right| \geq \lambda_{j} \\ 0, & \text { if }\left|d_{j, k}\right|<\lambda_{j} .\end{cases}
$$

The threshold $\lambda_{j}$ filters out the coefficients with value close to zero, allowing the significant coefficients related with the disturbance to pass. As most of the coefficients $d_{j, k}$ are close to zero, a valid criterion to select $\lambda_{j}$ is the use of the root-mean-square value at each level of $j$; thus,

$$
\lambda_{j}=\sqrt{\left(\frac{1}{K} \sum_{1}^{K}\left(d_{j, k}\right)^{2}\right)}, \quad k=1,2, \cdots, K
$$

where $K$ is the number of coefficients at level $j$.

When the algorithm executes this second step, a new reconstructed signal is obtained

$$
\tilde{a}_{0}(n)=a_{m}(n)+\sum_{j=1}^{m} \tilde{d}_{j}(n)
$$

where the level $m$ depends on the sampling frequency.

In both steps, the optimal relative error (ORE) between the input $a_{0}(n)$ and the reconstructed signal $\tilde{a}_{0}(n)$

$$
\mathrm{ORE}=\frac{\left\|a_{0}(n)-\tilde{a}_{0}(n)\right\|}{\left\|a_{0}(n)\right\|}
$$

is used for measuring the quality of the estimator.

In this method, we find a higher compression ratio and lower

\begin{tabular}{|c|c|c|}
\hline P & C & \\
\hline $\begin{array}{l}\text { momentary } \\
\text { interruption }\end{array}$ & 1 & \\
\hline momentary sag & 2 & \\
\hline instantaneous & 3 & \\
\hline instantaneous sag & 4 & 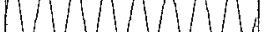 \\
\hline power frequency & 5 & \\
\hline momentary swell & 6 & \\
\hline $\operatorname{transient}$ & 7 & 1110 \\
\hline flicker & 8 & 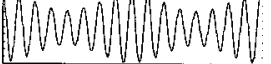 \\
\hline
\end{tabular}
ORE.

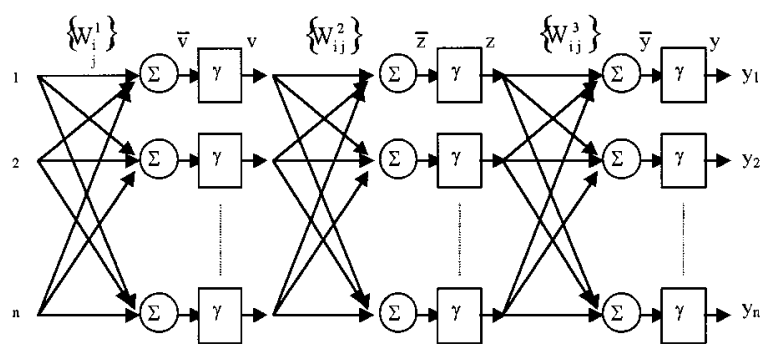

Fig. 4. Three-layer neural network.

TABLE I

Figures of Electrical Disturbances (P) TO BE MAPPED INTO CORRESPONDING Classes (C)

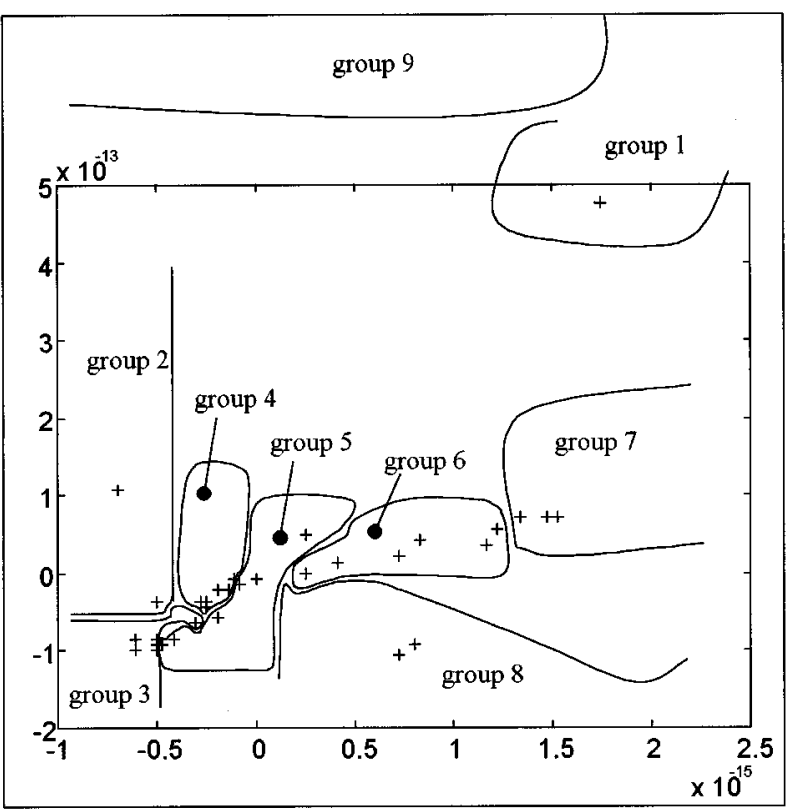

Fig. 5. Map of input patterns (disturbances). 
2) Classification: We select from among the $j$ levels of coefficients $d_{j, k}$ that $J$ with the highest energy content. This is essential for classifying the disturbance and localizing its timespan. At the same time, coefficients $d_{J, k}$ are compared with the equivalent coefficients of a previously stored sinusoidal signal $d_{J, k}^{\prime}$ which has followed the same process as the input signal. The result is a vector whose elements are differences of coefficients

$$
\text { vector }_{k}=d_{J, k}^{\prime}-d_{J, k}
$$

where $k$ is the coefficient order for the selected level of maximum energy. The components of vector (14) will be different from zero in the field where the signal disturbance exits.

The vector elements are used as inputs (input vector) of a learning vector quantization (LVQ) neural network with competitive learning in order to obtain stability during identification (Fig. 4). This three-layered LVQ network [9] automatically classifies eight types of electrical disturbance (Table I), associating each disturbance with one and only one of the set.

A representation of input patterns (disturbances) obtained by (14) is given in Fig. 5. These are the incoming vectors to be classified, and their coefficients are normalized to a two-dimensional (2-D) form for their graphical representation, using the "plotvec" function of MATLAB.

As we can see in Table I, there are eight types of disturbance to train, but they are grouped into nine different zones. As is well known, it is desirable to minimize the number of groups and the number of different perturbations belonging to each group, in order to obtain a minimum number of LVQ networks and a set of simplest training, thereby avoiding problems due to linear separability. In accordance, training sets formed by the input/output values are grouped in nine types of sets to train nine LVQ networks. The outputs of each LVQ network are $1 \times 1$ vectors representing the corresponding class for such disturbance.

As a result, the classification block consists of a set of nine LVQ networks with an output vector $\left(G_{1} \cdots G_{9}\right)$. The elements $G_{i}$ of the output vector of the neural network block have values from 1 to 8 , depending on the class of the recognized disturbance. If a disturbance is not recognized by the corresponding group, its value will be set to 10. (Fig. 6). Each LVQ network is specialized in recognizing a single group of disturbances. Only one LVQ network will give a valid output as the result of the classification, according to the classes defined in Table I.

To use the described architecture, once we have obtained the vector of a disturbance, it is presented to the whole neural network classification block, feeding each LVQ network to process the same vector at the same time.

To achieve parallel processing of the incoming disturbance toward the classification block, the vectors used in the training sets are selected from the boundaries between adjacent groups. Some vectors belonging to the inside zone of the group are also used.

The training set for all the LVQ networks use these vectors, but it is only set a valid output class in the LVQ network which will be specialized in the recognition of the disturbance (or sets of disturbances if there are more than two different ones in the same group). The other outputs are set to 10 , indicating a nonrecognized class.

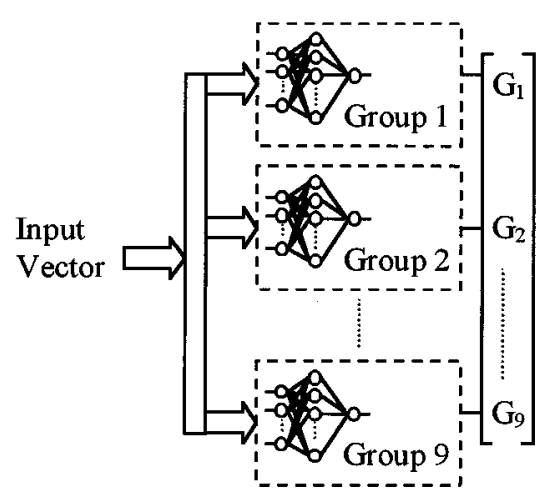

Fig. 6. Global architecture of the neural network block.

TABLE II

TRAINING SET FOR EACH LVQ NETWORK

\begin{tabular}{|c|c|c|c|c|c|}
\hline I & $\mathbf{D}$ & Group & $\mathbf{G}$ & Train/Test & success \\
\hline $\mathrm{kb} 2$ & $\begin{array}{l}\text { momentary } \\
\text { interruption }\end{array}$ & 1 & $\overline{1}$ & $35 / 70$ & $99 \%$ \\
\hline $\begin{array}{l}\text { kb10, kb30, } \\
\text { kb28, kb29 }\end{array}$ & $\begin{array}{c}\text { power } \\
\text { frequency } \\
\text { variation }\end{array}$ & 2 & 5 & $24 / 70$ & $97 \%$ \\
\hline $\begin{array}{c}\text { kb1, kb5, kb13, } \\
\text { kb14, kb20, kb21 }\end{array}$ & $\begin{array}{l}\text { momentary } \\
\text { interruption }\end{array}$ & 3 & 1 & $56 / 70$ & $74 \%$ \\
\hline $\begin{array}{l}\mathrm{kb} 11, \mathrm{~kb} 25, \\
\mathrm{~kb} 26, \mathrm{~kb} 27\end{array}$ & $\begin{array}{c}\text { momentary } \\
\text { swell }\end{array}$ & 4 & 6 & $21 / 70$ & $45 \%$ \\
\hline $\mathrm{kb} 10, \mathrm{~kb} 30$ & $\begin{array}{c}\text { power } \\
\text { frequency } \\
\text { variation }\end{array}$ & 4 & 5 & $34 / 70$ & $76 \%$ \\
\hline $\begin{array}{c}\text { kb24, kb6, kb8, } \\
\text { kb9 }\end{array}$ & $\begin{array}{c}\text { momentary } \\
\text { sag }\end{array}$ & 5 & 2 & $33 / 70$ & $56 \%$ \\
\hline $\begin{array}{c}\text { kb7, kb18, kb32, } \\
\text { kb35 }\end{array}$ & $\begin{array}{c}\text { instantaneous } \\
\text { sag }\end{array}$ & 6 & 4 & $52 / 70$ & $51 \%$ \\
\hline$\overline{\mathrm{kb}} 28, \mathrm{~kb} 29$ & $\begin{array}{c}\text { power } \\
\text { frequency } \\
\text { variation }\end{array}$ & 6 & 5 & $43 / \overline{70}$ & $\overline{84 \%}$ \\
\hline $\begin{array}{c}\text { kb3, kb19, kb31 } \\
\text { kb34 }\end{array}$ & $\begin{array}{l}\text { instantaneous } \\
\text { interruption }\end{array}$ & 7 & 3 & $81 / 70$ & $96 \%$ \\
\hline $\begin{array}{c}\mathrm{kb} 12, \mathrm{~kb} 15 \\
\mathrm{~kb} 16, \mathrm{~kb} 54, \mathrm{kb33}\end{array}$ & $\overline{\text { transient }}$ & 8 & 7 & $21 / 70$ & $75 \%$ \\
\hline $\mathrm{kb} 55$ & transient & 9 & 7 & $30 / 70$ & $91 \%$ \\
\hline $\begin{array}{l}\mathrm{kb} 39, \mathrm{~kb} 44 \\
\mathrm{~kb} 37, \mathrm{~kb} 38 \\
\mathrm{~kb} 36, \mathrm{~kb} 43\end{array}$ & flicker & 9 & $\overline{8}$ & $65 / 70$ & $94 \%$ \\
\hline
\end{tabular}

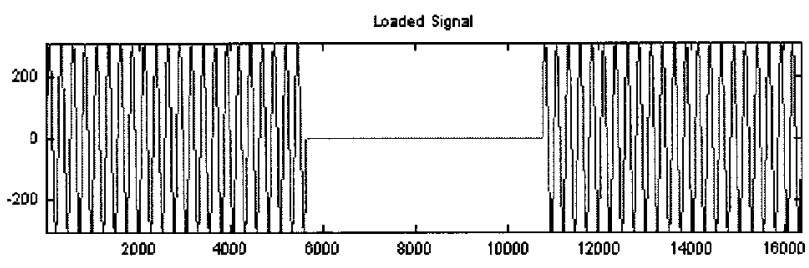

Fig. 7. Momentary voltage interruption (input signal).

In Table II, we can see those training sets. Inputs $(I)$ areincoming vectors of disturbances $(D)$. Outputs of the net $(G)$ are the classes to be mapped. In order to simplify the tables, we represent only 
some values used as recognized disturbances for each group. $K b i$ represents a coefficient vector obtained by a wavelet processing of the electrical disturbance. The numbers of training sets and test sets used are indicated in the last column of the table.

In order to define the evaluation criteria, we must consider that each LVQ network has been mapped to a different group of classes which contains one or more different disturbances, and also mapped to a code of nonrecognized disturbance (10). We collect the outputs of each block forming a $9 \times 1$ vector of nine artificial neural network (ANN) outputs. The output is correct if there is only one element different from code 10, and such output belongs to a class mapped by the LVQ network.

\section{B. Simulation Results}

The MATLAB program is used to calculate the DWT for two digitized signals, each with 16384 sample points. Figs. 7 and 14 show both signals used in the simulation: a momentary voltage interruption and a disturbed signal with two transients, respectively. A display of all the sampled points for a given "window" containing two transients cannot distinguish clearly two recorded disturbances. In the studied example, we show only the first 2000 sampled points contained in the first transient, instead of the input signal. The same is done with the simulation results. The data are sampled at 12800 samples per second. Figs. 8 and 15 show the decomposition of the above signals into $a_{j}(n)$ and $d_{j}(n)$ respectively, according to Section II. In our case, for $j=14$, the number of coefficients and frequency bands, at each level of $j$, are shown in Table III.

1) Momentary Voltage Interruption: In order to recover and compress the input signal, thresholding of wavelet transform coefficients can be performed according to the proposed method. Thus, by setting $\eta=0.03$, the initial threshold $\lambda=9.33$ is $3 \%$ of the maximum value of input signal, and condition (8) is fulfilled for $j=3\left(\max \left|\xi_{j}\right|=4.052\right)$. The smaller the ORE, the higher the quality of the reconstructed signal. In our case, ORE $=4.87 \cdot 10^{-4}$, which means that a good reconstruction of the signal is obtained, as shown in Fig. 9. The result and the data to be stored is only 2054 (Table III), which corresponds to a compression ratio $\tau=12.53 \%$.

The 13th and 14th levels are not shown because their frequency bands are a fraction of a hertz.

The differences between the input and reconstructed signal (error signal) are shown in Fig. 10.

The detail coefficient level $\left(d_{j, k}\right)$ of the higher energy level is $d_{8, k}$, which is shown in Fig. 11 for the sinusoidal signal and in Fig. 12 for the example signal.

The input vector obtained from (14) is shown in Fig. 13.

2) Transients: In this case, there is no level $j(\lambda=9.33$ and $\max \left|\xi_{1}\right|=9.58$ ) that fulfills condition (8), so the algorithm goes to the second step. We find a compromise between a good approximation for the input and reconstructed signal and a high rate of compression in the fourth level. For the considered levels, thresholds of $\lambda_{1}=0.220, \lambda_{2}=0.701, \lambda_{3}=7.039$, and $\lambda_{4}=5.720$ were found. The numbers of nonzero elements in signals $\tilde{d}_{1}(n), \tilde{d}_{2}(n), \tilde{d}_{3}(n)$, and $\tilde{d}_{4}(n)$ are $61,48,27$, and 24 respectively. To obtain $\tau$ we store the position and magnitude of modified coefficients at each level for the compressed signal,

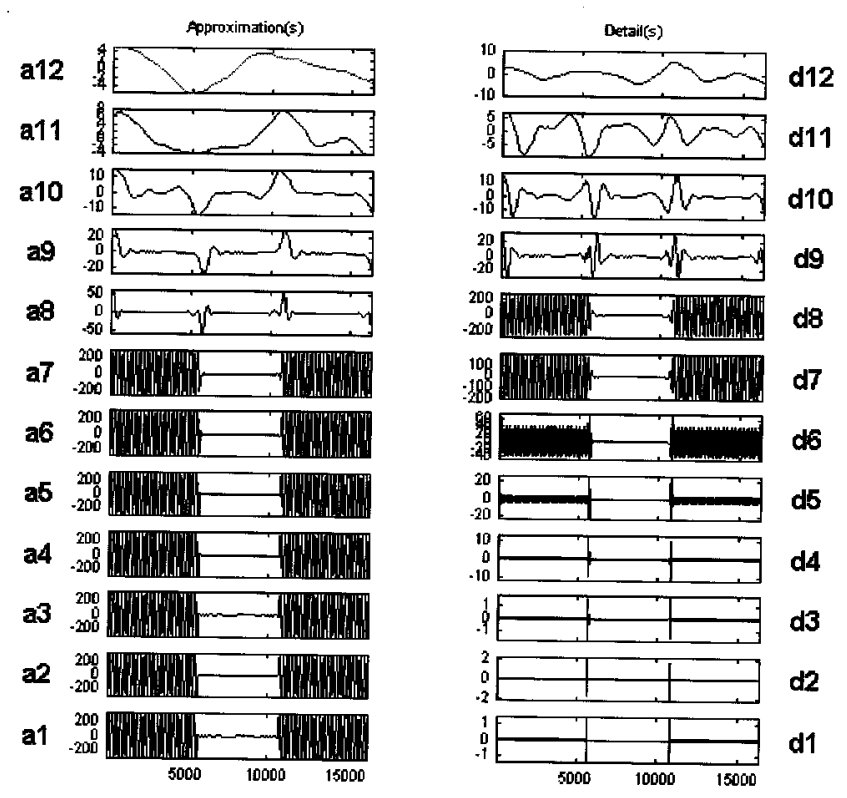

Fig. 8. Wavelet decomposition corresponding to Fig. 7.

TABLE III

NUMBER OF COEFFICIENTS AND FREQUENCY BAND

\begin{tabular}{|c|c|c|c|}
\hline & $\mathbf{a}_{j k}$ & $\begin{array}{l}\text { number of } \\
\text { coefficients }\end{array}$ & $d_{j k}$ \\
\hline level 1 & $0-3200 \mathrm{~Hz}$ & 8195 & $3200-6400 \mathrm{~Hz}$ \\
\hline level 2 & $0-1600 \mathrm{~Hz}$ & 4101 & $1600-3200 \mathrm{~Hz}$ \\
\hline level 3 & $0-800 \mathrm{~Hz}$ & 2054 & $800-1600 \mathrm{~Hz}$ \\
\hline level 4 & $0-400 \mathrm{~Hz}$ & 1030 & $400-800 \mathrm{~Hz}$ \\
\hline level 5 & $0-200 \mathrm{~Hz}$ & 518 & $200-400 \mathrm{~Hz}$ \\
\hline level 6 & $0-100 \mathrm{~Hz}$ & 262 & $100-200 \mathrm{~Hz}$ \\
\hline level 7 & $0-50 \mathrm{~Hz}$ & 134 & $50-100 \mathrm{~Hz}$ \\
\hline level 8 & $0-25 \mathrm{~Hz}$ & 70 & $25-50 \mathrm{~Hz}$ \\
\hline level 9 & $0-12,5 \mathrm{~Hz}$ & 38 & $12,5-25 \mathrm{~Hz}$ \\
\hline level 10 & $0-6,25 \mathrm{~Hz}$ & 22 & $6,25-12,5 \mathrm{~Hz}$ \\
\hline level 11 & $0-3,125 \mathrm{~Hz}$ & 14 & $3,125-6,25 \mathrm{~Hz}$ \\
\hline level 12 & $0-1,56 \mathrm{~Hz}$ & 10 & $1,56-3,125 \mathrm{~Hz}$ \\
\hline level 13 & $0-0,78 \mathrm{~Hz}$ & 8 & $0,78-1,56 \mathrm{~Hz}$ \\
\hline level 14 & $0-0,39 \mathrm{~Hz}$ & 7 & $0,39-0,78 \mathrm{~Hz}$ \\
\hline
\end{tabular}

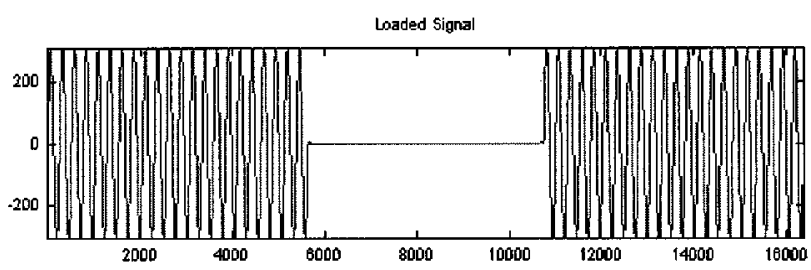

Fig. 9. Reconstructed signal of Fig. 7.

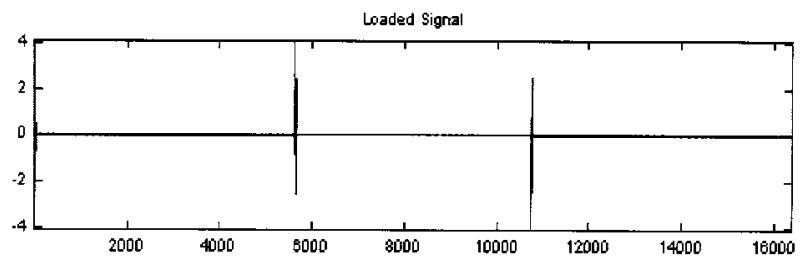

Fig. 10. Error signal. 


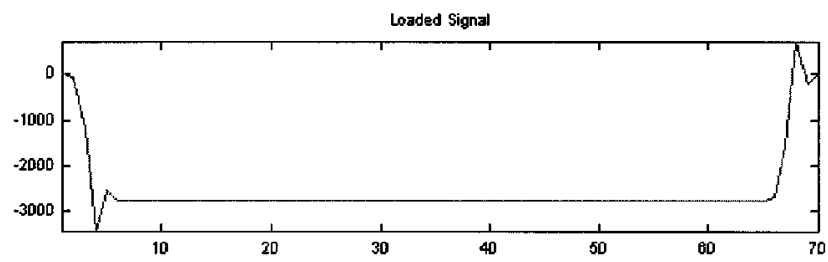

Fig. 11. $d_{8, k}$ coefficients of the sinusoidal signal.

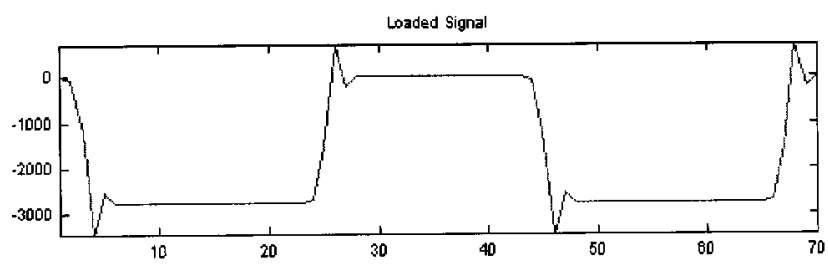

Fig. 12. $d_{8, k}$ coefficients of the momentary voltage interruption

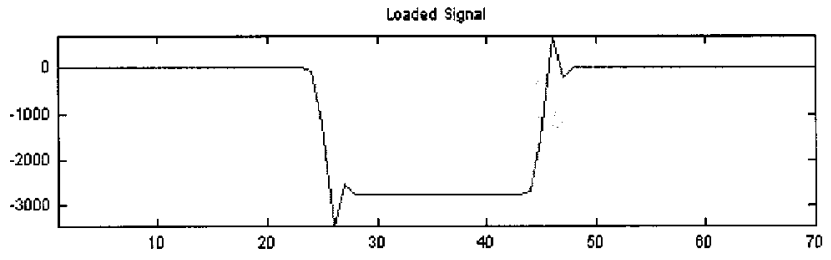

Fig. 13. Input vector.

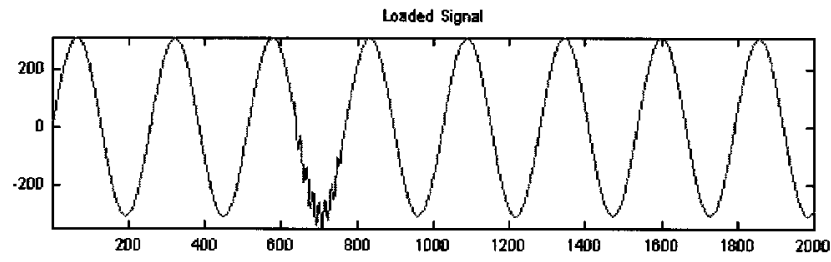

Fig. 14. Transients (input signal)

so 1350 data $(61 \times 2+48 \times 2+27 \times 2+24 \times 2+1030)$ are necessary.

The input signal is shown in Fig. 14 and its wavelet decomposition in Fig. 15.

The reconstructed signal is shown in Fig. 16, and the difference between the input and reconstructed signals (error signal) in Fig. 17. The ORE is 0.0191 , and $\tau=8.24 \%$. Thus, although in this case the ORE is higher, the rate of compression is improved.

The level of the higher energy is also $d_{8, k}$, (Fig. 18), and the input vector is shown in Fig. 19.

Table IV shows the results of several simulations. Each column represents the output vector of the neural network block, and we can see that the element $G_{i}$, that is, the output of group $i$, recognizes the corresponding disturbance. In particular, we can note the results obtained using the two disturbances described in the paper (momentary interruption, kb22 shown in Fig. 13 and transient, kb17 shown in Fig. 19).
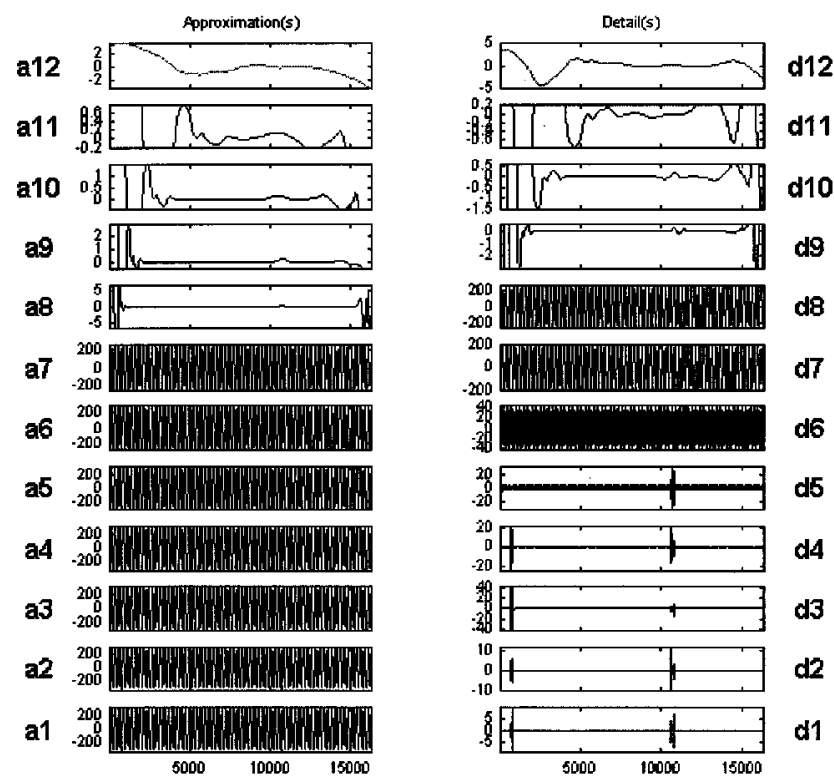

Fig. 15. Wavelet decomposition of Fig. 14.

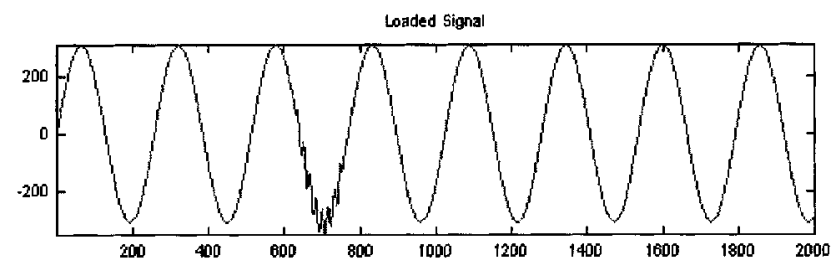

Fig. 16. Reconstructed signal of Fig. 14.

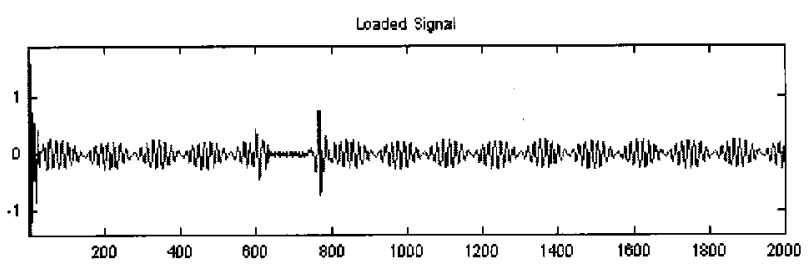

Fig. 17. Error signal.

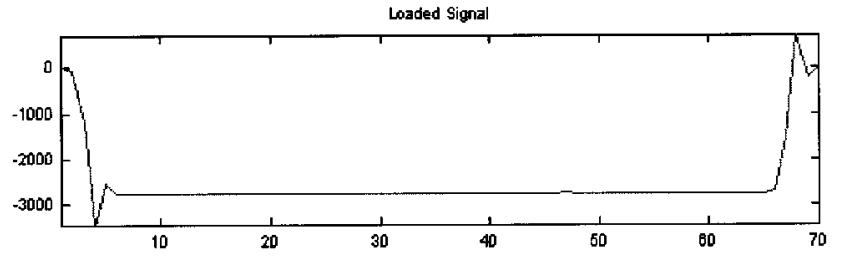

Fig. 18. $d_{8, k}$ coefficients of the transients.

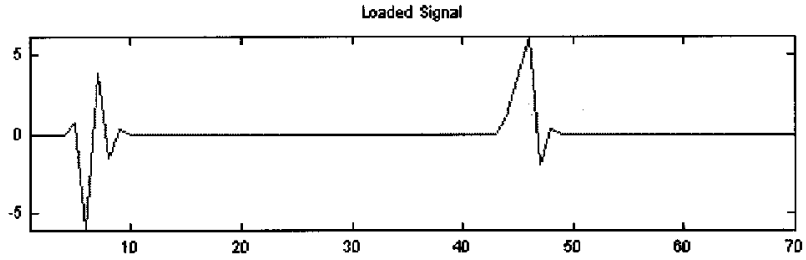

Fig. 19. Input vector. 
TABLE IV

OutPut Vector CORRESPONDING To SEVERAL Simulations

\begin{tabular}{|c|c|c|c|c|c|c|c|c|c|}
\hline & & & & & & & & \\
\hline & & & & & & \\
\hline
\end{tabular}

\section{CONCLUSION}

The most relevant and typical disturbances in electrical power systems can be analyzed by means of the method proposed in this paper. Furthermore, we study data compression and propose an optimal solution in the wavelet domain. A neural classification system using wavelet analysis has been used to distinguish eight typical disturbances.

This work leads us to believe that wavelet analysis together with neural structure, as a new tool, offers a great potential for diagnosis of electrical power systems in the area of power quality problems.

\section{REFERENCES}

[1] J. C. Montaño, M. Castilla, J. Gutiérrez, and A. López, "Sistema de medida y vigilancia de la calidad del suministro de potencia eléctrica," in Proc. Sas Jornadas Hispano-Lusas de Ingeniería Eléctrica, Salamanca, Spain, July 1997, pp. 1643-1650.

[2] C. Roberton, O. I. Camps, J. S. Mayer, and W. B. Gish, "Wavelet and electromagnetic power system transients," IEEE Trans. Power Delivery, vol. 11, pp. 1050-1058, Apr. 1996.

[3] S.-J. Huang et al., "Application of Morlet wavelet to supervise power system disturbances," IEEE Trans. Power Delivery, vol. 14, pp. 235-245, Jan. 1999.

[4] H. Guo, M. Lang, J. E. Odegard, and C. S. Burrus, "Nonlinear shrinkage of undecimated DWT for noise reduction and data compression," in Proc. Int. Conf. Digital Signal Processing, Limassol, Cyprus, June 1995, pp. 332-337.

[5] S. Mallat, "A theory for multiresolution signal decomposition: the wavelet representation," IEEE Trans. Pattern Anal. Machine Intell., vol. 11, pp. 674-693, July 1989.

[6] C. Sidney Burrus, R. A. Gopinath, and H. Guo, Introduction to Wavelets and Wavelet Transforms. Englewood Cliffs, NJ: Prentice-Hall, 1998, p. 7458.

[7] W. A. Wilkinson and M. D. Cox, "Discrete wavelet analysis of power system transients," IEEE Trans. Power Syst., vol. 11, pp. 2038-2044, Nov. 1996.

[8] S. Santoso and P. Hofmann, "Power quality assessment via wavelet transform analysis," IEEE Trans. Power Delivery, vol. 11, pp. 924-930, Apr. 1996.

[9] T. Kohonen, "The self-organizing map," in Neural Networks: Theoretical Foundations and Analysis. New York: IEEE Press, 1991, pp. 74-90.

[10] D. L. Donoho and I. M. Johnstone, "Ideal spatial adaptation by wavelet shrinkage," Dep. Statistics, Stanford Univ., Stanford, CA, Tech. Rep. 400, July 1992

[11] N. Kandil, V. K. Sood, K. Khorasani, and R. V. Patel, "Fault identification in AC-DC transmission system using neural networks," IEEE Trans. Power Syst., vol. 7, pp. 812-819, May 1992.

[12] S. Kumar Pandey and L. Satish, "Multiresolution signal decomposition: A new tool for fault detection in power transformers during impulse test," IEEE Trans. Power Delivery, vol. 13, pp. 1194-1200, Oct. 1998.

[13] R. K. Hartana and G. G. Richards, "Constrained neural network-based identification of harmonic sources," IEEE Trans. Ind. Applicat., vol. 29, pp. 202-208, Jan./Feb. 1993

[14] D. L. Donoho, I. M. Johnstone, G. Kerkyacharian, and D. Picard, "Density estimation by wavelet thresholding," Ann. Statist., vol. 24, pp. 508-539, 1996.

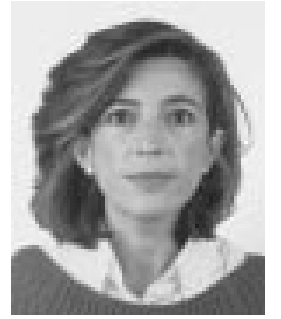

Dolores Borrás was born in Seville, Spain, in 1966. She received the "Licenciado" degree in physics sciences from the University of Seville, Seville, Spain, in 1990.

Since 1992, she has been a full-time Professor of Electrical Circuits and Power Electronics in the Polytechnic School, Department of Electrical Engineering, University of Seville. Her research interests include digital signal processing and signal analysis via wavelet transform.

Prof. Borrás is a member of the Invespot Research Group on Electrical and Electronic Measurements.

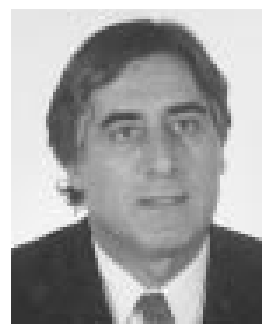

M. Castilla (M'94) was born in Seville, Spain, in 1944. He received the "Licenciado" and Ph.D. degrees in physics sciences from the University of Seville, Seville, Spain.

From 1968 to 1982, he was a Professor of Circuits Theory and Power Electronics at the Polytechnic Institute of La Rábida, Huelva, Spain. Since 1983, he has been a Professor in the Electrical Engineering Department, Polytechnic School, University of Seville. His research has been devoted to analysis and measurement of apparent power components in nonsinusoidal conditions, power transfer quality in nonsinusoidal situations, and assessment via wavelet transform analysis. He has participated in different projects related to power quality.

Dr. Castilla is a member of the Invespot Group on Electrical and Electronic Measurements.

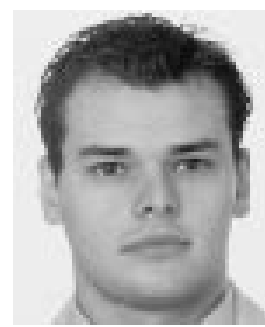

trol techniques.

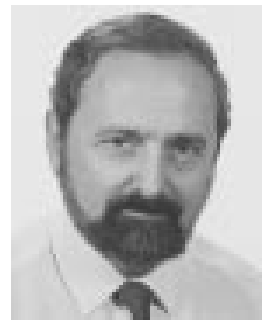

J. C. Montaño (M'80-SM'00) was born in Sanlucar de Barrameda, Spain, in 1940. He received the Ph.D. degree in physics from the University of Seville, Seville, Spain, in 1972

In 1973, he joined the Instituto de Automatica Industrial, Consejo Superior de Investigaciones Científicas (CSIC), Madrid, Spain, where he was a Researcher until 1978, working on analog signal processing, electrical measurements in power systems, and control of industrial processes. Since 1978, he has been in charge of various projects in connection with research on power theory of nonsinusoidal systems and reactive power control in electrical systems at the CSIC in Seville, Spain. At present, he is leading a project for monitoring power quality.

Dr. Montaño is a member of the Invespot Group on Electrical and Electronic Measurements. 